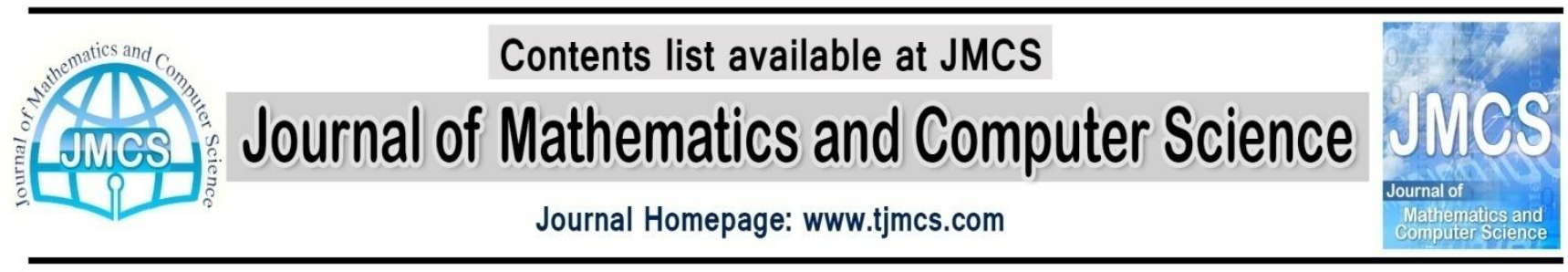

\title{
Hyers-Ulam Stability of Fibonacci Functional Equation in Modular Functional Spaces
}

\author{
Maryam Naderi Parizi ${ }^{1}$, Madjid Eshaghi Gordji ${ }^{2, *}$ \\ ${ }^{1}$ Department of Mathematics, Payame noor University, Tehran, Iran \\ ${ }^{2}$ Department of Mathematics, Semnan University ,P.O.BOX35195-363, Semnan. Iran \\ E-mail \\ mrym_ndr@yahoo.com ${ }^{1}$, madjid.eshaghi@gmail.com² \\ *The corresponding author (M. E. Gordji)
}

Received January 2014

Accepted March 2014

Available online March 2014

Abstract

In this paper, we prove the Hyers-Ulam stability of functional equation

$$
f(x)=f(x-1)+f(x-2)
$$

which called the Fibonacci functional equation in modular functional space.

Keywords: Hyers-Ulam stability, Fibonacci functional equation, modular functional space.

\section{Introduction}

In 1950 Nakano [16] introduced the theory of modular spaces in connection with the theory of ordered spaces, Musielak and Orlicz [15] in 1959, redefined and generalized it to obtain a generalization of the classical function spaces $L^{\wedge} p$.

Also the stability of functional equations originated from a question of Ulam [19] in 1940. In the next year, Hyers [9] proved the problem for the Cauchy functional equation. The stability problems of functional equations have been extensively investigated by several mathematician (see $[3,4,5,6,7,8,10,11,12,14,17]$ and [18]).

Recently, Jung [13] investigated the Hyers-Ulam stability of Fibonacci functional equation $f(x)=f(x-$ $1)+\mathrm{f}(\mathrm{x}-2)$.Here we prove the Hyers-Ulam stability of this functional equation in modular functional space. By $\alpha$ and $\beta$ we denote roots of the equation $x^{2}-x-1=0 . \alpha+\beta=1 \alpha$ is greater than one and $\beta$ is negative root. We have $\alpha+\beta=1$ and $\alpha \beta=-1$. 


\section{Preliminaries}

We recall some basic notions and facts about Modular spaces.

Definition2.1: Let $X$ be an arbitrary vector space over a complex or real field.

(a) A function $\rho: X \rightarrow[0,+\infty]$ is called a modular If

(i) $\rho(x)=0 \Leftrightarrow x=0$,

(ii) $\rho(\alpha x)=\rho(x)$ for every scaler $|\alpha|$ with $|\alpha=1|$,

(iii) $\rho(\alpha x+\beta y) \leq \rho(x)+\rho(y) \quad \alpha+\beta=1, \alpha \geq 0, \beta \geq 0$

for all $x, y \in \mathbf{R}$.

(b) If (iii) is replaced by

$\rho(\alpha x+\beta y) \leq \alpha \rho(x)+\beta \rho(y)$ if $\alpha+\beta=1$

And $\alpha \geq 0, \beta \geq 0$ we say that $\rho$ is convex modular.

(c) A modular $\rho$ defines a corresponding modular space, i.e.the vector space $X_{\rho}$ given by $X_{\rho}=\{x \in X: \rho(\lambda x) \rightarrow 0$ as $\lambda \rightarrow 0\}$

Example2.2: $\operatorname{Let}(X,\|\|$.$) be a norm space, then \|$.$\| is a convex modular$ on $\mathrm{X}$. But the converse is not true.

In general the modular $\rho$ does not behave as a norm or a distance because it is not sub--additive. But one can associate to a modular the $\mathrm{F}$-norm (see [9]).

Definition2.3: The modular space $X_{\rho}$ can be equipped with the F-norm defined by

$$
|x|_{\rho}=\inf \left\{\alpha>0 ; \rho\left(\frac{x}{\alpha}\right) \leq \alpha\right\}
$$

Namely, if $\rho$ be convex, then the functional

$$
\|x\|_{\rho}=\inf \left\{\alpha>0 ; \rho\left(\frac{x}{\alpha}\right) \leq 1\right\}
$$

is a norm called the Luxemburg norm in $X_{\rho}$ which is equivalent to the $|.|_{\rho}$.

Definition2.4: Let $X_{\rho}$ be a modular space.

(a) A sequence $\left\{x_{n}\right\}_{n \in \square}$ in $X_{\rho}$ is said to be:

(i) $\rho$ - convergent to $x$ if $\rho\left(x_{n}-x\right) \rightarrow 0$ as $n \rightarrow \infty$;

(ii) $\rho$-Cauchy if $\rho\left(x_{n}-x_{m}\right) \rightarrow 0$ as $n, m \rightarrow \infty$;

(b) $X_{\rho}$ is $\rho$-complete if every $\rho$-Cauchy sequence

is $\rho$ - convergent.

(c) A subset $B \subseteq X_{\rho}$ is said to be $\rho$-closed if for any sequence $\left\{x_{n}\right\}_{n \in \square} \subset B$ with $x_{n} \rightarrow x$, then $x \in B$.

(d) A subset $B \subseteq X_{\rho}$ is called $\rho$-bounded if 


$$
\delta_{\rho}(B)=\sup \{\rho(x-y): x, y \in B\}<\infty
$$

where $\delta_{\rho}(B)$ is called the $\rho$-diameter of B .

(e) We say that $\rho$ has the Fatou property if

$$
\rho(x-y) \leq \underline{\lim } \rho\left(x_{n}-y_{n}\right)
$$

whenever $\rho\left(x_{n}-x\right) \rightarrow 0, \rho\left(y_{n}-y\right) \rightarrow 0$ as $n \rightarrow \infty$.

(f) $\rho$ is said to satisfies the $\Delta_{2}$-condition if

$\rho\left(2 x_{n}\right) \rightarrow 0$ as $n \rightarrow \infty$ whenever $\rho\left(x_{n}\right) \rightarrow 0$ as $n \rightarrow \infty$.

It is easy to check that for every modular $\rho$ and $x, y \in X_{\rho}$;

(1) $\rho(\alpha x) \leq \rho(\beta x)$ for each positive numbers with $\alpha \leq \beta$.

(2) $\rho(x+y) \leq \rho(2 x)+\rho(2 y)$.

\section{Hyers-Ulam Stability of Fibonacci equation in modular space}

As already stated, $\alpha$ denotes the positive root of the equation $x^{2}-x-1=0$ and $\beta$ is its negetive root. We can prove the Hyers-Ulam stability of the Fibonacci functional equation (1.1) as we see in the following theorem.

Theorem3.1: Let $(X, \rho)$ be a Banach modular space. If $\mathrm{f}: \mathbf{R} \rightarrow X$ satisfies the inequality

$\rho(f(x)-f(x-1)-f(x-2)) \leq \varepsilon$

for all $x \in \mathbf{R}$ and for some $\varepsilon>0$ then there exists a Fibonacci function $\mathrm{G}: \mathbf{R} \rightarrow \mathrm{X}$ such that

$\rho(f(x)-G(x)) \leq\left(1+\frac{2}{\sqrt{5}}\right) \varepsilon$

for all $x \in \mathbf{R}$.

Proof.We get from (3.1):

$\rho(f(x)-\alpha f(x-1)-\beta[f(x-1)-\alpha f(x-2)]) \leq \varepsilon$

If we replace $x$ by $x-k$ in the last inequality, then we have,

$\rho(f(x-k)-\alpha f(x-k-1)-\beta[f(x-k-1)-\alpha f(x-k-2)]) \leq \varepsilon$

and furthermore,

$$
\begin{aligned}
& \rho\left(\beta ^ { k } \left(f(x-k)-\alpha f(x-k-1)-\beta^{k+1}(f(x-k-1)-\alpha f(x-k-2))\right.\right. \\
& \leq\left|\beta^{k}\right| p(f(x-k)-\alpha f(x-k-1)-\beta[f(x-k-1)-\alpha f(x-k-2)]) \\
& \leq\left|\beta^{k}\right| \varepsilon
\end{aligned}
$$

for all $x \in \mathbf{R}$ and natural number k. By (3.3), we obviously have,

$\rho\left(f(x)-\alpha f(x-1)-\beta^{n}[f(x-n)-\alpha f(x-n-1)]\right)$

$=\rho\left(\sum_{k=0}^{n-1} \beta^{k}\left(f(x-k)-\alpha\left(f(x-k-1)-\beta^{k+1}(f(x-k-1)-\alpha f(x-k-2))\right.\right.\right.$ 


$$
\begin{aligned}
& \leq \sum_{k=0}^{n-1}|\beta|^{k} \rho((f(x-k)-\alpha f(x-k-1)-\beta(f(x-k-1)-\alpha f(x-k-2))) \\
& \leq \sum_{k=0}^{n-1}|\beta|^{k} \text { ò } \leq \frac{\mathrm{ò}}{1-|\beta|}
\end{aligned}
$$

for all $x \in \mathbf{R}$ and natural number $\mathrm{n}$.

For any $x \in \mathbf{R}(3.3)$ implies that $\left\{\beta^{n}(f(x-n)-\alpha f(x-n-1))\right\}$ is a cauchy sequence .

Therefore, we can define a function by

$$
G_{1}(x)=\lim _{n \rightarrow \infty} \beta^{n}[f(x-n)-\alpha f(x-n-1)]
$$

since $\mathrm{X}$ is complete. In view of the above definition of $\mathrm{G}_{1}$, we obtain:

$$
\begin{aligned}
& G_{1}(x-1)+G_{1}(x-2)=\beta^{-1} \lim _{n \rightarrow \infty} \beta^{n+1}[f(x-(n+1))-\alpha f(x-(n+1)-1)] \\
& +\beta^{-2} \lim _{n \rightarrow \infty} \beta^{n+2}[f(x-(n+2)-\alpha f(x-(n+2)-1)] \\
& =\beta^{-1} G_{1}(x)+\beta^{-2} G_{1}(x)=G_{1}(x)
\end{aligned}
$$

for all $x \in \mathbf{R}$. Hence, $\mathrm{G}_{1}$ is a Fibonacci function. If $\mathrm{n}$ goes to infinity, then (3.3) yields:

$$
\rho\left(f(x)-\alpha f(x-1)-G_{1}\right) \leq \frac{3+\sqrt{5}}{2} \varepsilon
$$

for all $x \in \mathbf{R}$.on the other hand, it also follows from (3.1) that

$\rho(f(x)-\beta f(x-1)-\alpha[f(x-1)-\beta f(x-2)]) \leq \varepsilon$

Analogous to (3.3), replacing $x$ by $x+k$ in the above

Inequality, then we have:

$\rho(f(x+k)-\beta[f(x+k-1)-\alpha f(x+k-1)-\beta f(x+k-2)]) \leq \varepsilon$

and

$\rho\left(\alpha^{-k}[f(x+k)-\beta f(x+k-1)]-\alpha^{-k+1}[f(x+k-1)-\beta f(x+k-2)]\right) \leq|\alpha|^{-k} \varepsilon$

for all $x \in \mathbf{R}$. By using (3.6), we further obtain:

$\rho\left(\alpha^{-n}[f(x+n)-\beta f(x+n-1)]-[f(x)-\beta f(x-1)]\right)$

$\leq \sum_{k=1}^{n} \rho\left(\alpha^{-k}[f(x+k)-\beta f(x+k-1)]-\alpha^{-k+1}[f(x+k-1)-\beta f(x+k-2)]\right)$

$\leq \sum_{k=1}^{n} \alpha^{-k} \rho([f(x+k)-\beta f(x+k-1)]-\alpha[f(x+k-1)-\beta f(x+k-2)])$

$\leq \sum_{k=1}^{n} \alpha^{-k} \varepsilon$

for all $x \in \mathbf{R}$ and $\$$ nlin|BN\$. Thus $\left\{\alpha^{-n}[f(x+n)-\beta f(x+n-1)]\right\}$ is a cauchy sequence, for any fixed $x \in \mathbf{R}$. Hence, we can define a function by

$G_{2}(x)=\lim _{n \rightarrow \infty} \alpha^{-n}[f(x+n)-\beta f(x+n-1)]$

Using the above definition of $\mathrm{G}_{2}$, we get: 


$$
\begin{aligned}
& G_{2}(x-1)+G_{2}(x-2)=\alpha^{-1} \lim _{n \rightarrow \infty} \alpha^{-(n-1)}[f(x+n-1)-\beta f(x+(n-1)-1)] \\
& +\alpha^{-2} \lim _{n \rightarrow \infty} \alpha^{-(n-2)}[f(x+n-2)-\beta f(x+(n-2)-1)] \\
& =\alpha^{-1} G_{2}(x)+\alpha^{-2} G_{2}(x)=G_{2}(x)
\end{aligned}
$$

For any $x \in \mathbf{R}$. So, $G_{2}$ is also a Fibonacci function. If we

Let $n$ goes to infinity, then it follows from (3.7) that

$$
\rho\left(G_{2}(x)-f(x)-\beta f(x-1)\right) \leq \frac{\sqrt{5}+1}{2} \varepsilon
$$

for $x \in \mathbf{R}$. By (3.5), (3.8) we have,

$$
\begin{aligned}
& \rho\left(f(x)-\left[\frac{\beta}{\beta-\alpha} G_{1}-\frac{\alpha}{\beta-\alpha} G_{2}\right]\right) \\
& \leq \frac{\alpha}{\alpha-\beta} \rho\left(f(x)-\beta f(x-1)-G_{2}\right)-\frac{\beta}{\alpha-\beta} \rho\left(f(x)-\alpha f(x-1)-G_{1}\right) \\
& =\rho\left(\frac{\alpha}{\alpha-\beta}\left(f(x)-\beta f(x-1)-G_{2}\right)-\frac{\beta}{\alpha-\beta}\left(f(x)-\alpha f(x-1)-G_{1}\right)\right) \leq\left(1+\frac{2}{\sqrt{5}}\right) \text { ò }
\end{aligned}
$$

for all $x \in \mathbf{R}$. We now set:

$G(x)=\frac{\beta}{\beta-\alpha} G_{1}-\frac{\alpha}{\beta-\alpha} G_{2}$

It is easy to show that $G$ is a Fibonacci function satisfying (3.2).

\section{References}

[1] M. Bidkham and M. Hosseini, Hyers-Ulam stability of \$k\$-Fibonaccifunctional equation, Internat. J. Nonlinear Anal. Appl. 2 (2011), 42--49.

[2] M. Bidkham, M. Hosseini, C. Park and M. Eshaghi Gordji, Nearly (k,s) --Fibonacci functional equations in beta--normed spaces, Aequationes Math. 83 (2012), 131--141.

[3] M. Eshaghi Gordji and H. Khodaei, Stability of Functional Equations, LAP LAMBERT Academic Publishing, 2010.

[4] G.L. Forti, Hyers-Ulam stability of functional equations in several variables, Aequationes Math. 50 (1995), 143--190.

[5] Z. Gajda, On stability of additive mappings, Internat. J. Math. Math. Sci. 14 (1991), 431--434.

[6] P. Gavrota, A generalization of the Hyers-Ulam-Rassias stability of approximately additive mappings, J. Math. Anal. Appl. 184 (1994), 431--436.

[7] P. Gavruta and L. Gavruta, A new method for the generalized Hyers-Ulam-Rassias stability, Int. J. Nonlinear Anal. Appl. 1 (2010),11--18.

[8] R. Ger and P. Semrl, The stability of the exponential equation, Proc. Amer. Math. Soc. 124 (1996), $779--787$.

[9] D.H. Hyers, On the stability of the linearfunctional equation, Proc. Natl. Acad. Sci. USA 27 (1941), 221--224.

[10] D.H. Hyers, G. Isac and Th.M. Rassias, Stability of Functional Equations in Several Variables, Birkhauser, Boston, 1998.

[11] D.H. Hyers and Th.M. Rassias, Approximate homomorphisms, Aequationes Math. 44 (1992), 125--153. 
M. Naderi Parizi, M. E. Gordji / J. Math. Computer Sci. 10 (2014), 1-6

[12] S. Jung, Hyers-Ulam-Rassias Stability of Functional Equations in Mathematical Analysis, Hadronic Press, Palm Harbor, 2001.

[13] S. Jung, Hyers-Ulam stability of Fibonacci functional equation, Bull. Iranian Math. Soc. 35 (2009), 217--227.

[14] S. Jung, Hyers--Ulam--Rassias stability of functional equations in nonlinear analysis, Springer Optimization and Its Applications, 48, Springer, New York, 2011.

[15] J. Musielak and W. Orlicz On modular spaces, Studia Mathematica.18 (1959), 49--65.

[16] H. Nakano, Modular semi-ordered spaces, Tokyo Mathematical Book Series, Maruzen Co. Ltd,Tokyo, Japan, (1950).

[17] Th.M. Rassias, On the stability of the linear mapping in Banach spaces, Proc. Amer. Math. Soc. 72 (1978), $297--300$.

[18] Th.M. Rassias, Approximate homomorphisms, Aequationes Math. 44 (1992), 125--153.

[19] S.M. Ulam, Problems in Modern Mathematics, Chapter VI, Science ed., Wiley, New York, 1940. 